\title{
Improvement of the reliability and durability parameters of hydrotechnical structures under conditions of hydrodynamic influence of flows on structural elements
}

\author{
Alexander Solovyev ${ }^{1,2}$, Dmitry Solovyev ${ }^{3}$ and Liubov Shilova, ${ }^{4, *}$ \\ ${ }^{1}$ M.V. Lomonosov Moscow State University, Faculty of Geography, 19991, 1/19 Lenin Hills, \\ Moscow, Russia \\ ${ }^{2}$ Moscow State Academy of Water Transport, 117105, 2/1 Novodanilovskaya nab., Moscow, Russia \\ ${ }^{3}$ Shirshov Institute of Oceanology, Russian Academy of Sciences, 117997, 36 Nahimovskiy prospekt, \\ Moscow, Russia \\ ${ }^{4}$ Moscow State University of Civil Engineering, Yaroslavskoe shosse, 26, Moscow, 129337, Russia
}

\begin{abstract}
This article concerns the methodological issues of developing principles for increasing the reliability and durability parameters of equipment and auxiliary mechanisms of hydrotechnical structures. An analysis of the conditions ensuring an increase in the accuracy of engineering calculations of the viscosity of disperse systems is carried out. An analytical consideration of the effects of the particle shape on the calculation of the groundwater viscosity using the representations of particles of dispersed media as hydrodynamic dipoles is proposed. An equation for the viscosities of disperse systems is obtained with the determination of the shape factor of solid particles in a liquid. The comparison with the ratios for calculating the viscosity of ideal groundwater consisting of particles of a circular configuration indicates the possibility of reducing errors in calculating the coefficients of permeability of flow rates and pressures of filtration currents in underground horizons under the fluences of hydraulic structures. To improve the reliability of building structural elements operation of spillways without vacuum dams, a method of refined calculation of the dynamic effect of groundwater on the base of spillway dams and dams of the river bed is proposed.
\end{abstract}

\section{Introduction}

The viscosity of inhomogeneous media consisting of solid particles in a liquid is one of the characteristics of groundwaters affecting the parameters of their filtration, which determine the spatial variation of the depression pressure in the underground contours of hydraulic structures. The effect of dispersed phase particles on the viscous flow of groundwater and on the spatial variation of subterranean pressure is due to the fact that those layers of the moving aquatic environment that adjoin directly to the particles move only at the same rate

*Corresponding author: ShilovaLA@mgsu.ru 
as water [1]. This effect was taken into account for spherical particles in [2]. Taking into account that at a sufficient distance from the surface of the particle the flow has a constant velocity, and the particle itself moves along with the flow, the following relation is obtained for the viscosity of the disperse system:

$$
\eta=\eta_{\mathrm{o}}(1+0,5 \phi)
$$

where $\eta$ is the viscosity of the inhomogeneous medium; $\phi$ - volume fraction of particles of the disperse phase; 0.5 is the coefficient of the shape of the spherical particles; $\eta_{\mathrm{o}}=\eta / \eta_{\mathrm{o}}$ - relative viscosity.

In the following, the refined dependence of the viscosity with non-spherical particles in filter flows with different intensity of pressure and different orientation of the particles to the direction of flow was considered. As a result, an equation was obtained for the specific viscosity of a dispersive medium showing the increment of viscosity relative to a dispersed medium $\eta^{*}$ in which the shape of the particles was taken into account through a certain numerical coefficient $\Phi[3]$.

$$
\eta^{*}=\left(\eta-\eta_{\mathrm{o}}\right) / \eta_{\mathrm{o}}=\Phi \phi,
$$

where $\Phi$ is the empirical coefficient of the shape of the particles with a numerical value exceeding the value $\Phi=0.5$ and which depends not only on the shape of the particles but also on the gradient of the flow velocity and the orientation of the particles, leading to resistance to flow and pressure losses.

In subsequent work, studies of the viscosity of inhomogeneous media were extended to the case of droplet particles capable of taking a non-spherical shape. Experimental checks of the calculated ratios for the viscosity of inhomogeneous media indicated significant discrepancies with the calculations, especially for media with deformable particles of nonideal form [4]. In fact, there are still no adequate formulas for calculating the viscosity of groundwater with non-ideal solid particles, as applied to project evaluations of the effects of filtration currents on hydraulic plant fluids, although work in this direction does not stop $[5 ; 7 ; 9]$. It remains an open question about how to take into account the change in the tangential viscosity of an inhomogeneous medium with respect to a liquid medium in determining the dynamic interactions of groundwater flows with elements of hydraulic structures.

\section{Viscosity of inhomogeneous medium}

Let us turn to the determination of the dependence of the viscosity of porous media such as groundwaters on the conditions of non-ideal-like flow past a liquid medium. We consider the inhomogeneous medium to be a medium composed of two constituents, different in their physicochemical properties of solid particles, distributed in the liquid as individual elements of a volume of a non-ideal form. We represent an inhomogeneous medium in the form of a system of hydrodynamic dipoles. The velocity potential of such dipoles is:

$$
\mathrm{F}=-\frac{\mathrm{Q}}{\mathrm{R}_{1}}+\frac{\mathrm{Q}}{\mathrm{R}_{2}}
$$

Where $R_{1}=\sqrt{(x-r)^{2}+y^{2}+z^{2}}, R_{2}=\sqrt{(x+r)^{2}+y^{2}+z^{2}}, Q$ - power of the source, $2 r$ is the length of the dipole.

The components of the flow velocity of a liquid medium with particles at an arbitrary point are equal to

$$
\mathrm{W}_{\mathrm{oi}}=\frac{\partial \mathrm{F}}{\partial \mathrm{x}_{\mathrm{i}}}=\mathrm{Q}\left[\frac{\alpha_{1 \mathrm{i}}}{\mathrm{R}_{1}^{2}}-\frac{\alpha_{2 \mathrm{i}}}{\mathrm{R}_{2}^{2}}\right]
$$


Here $\alpha_{1 \mathrm{i}}, \alpha_{2 \mathrm{i}}$ are the direction cosines of the vectors $\ddot{\mathrm{R}}_{1}, \ddot{\mathrm{R}}_{2}$.

From here one can obtain the following expressions for such quantities as:

-module of the velocity of the liquid medium;

$\mathrm{W}_{0}=\sqrt{\frac{2 \mathrm{Q}^{2}}{\mathrm{R}_{1}^{2} \mathrm{R}_{2}^{2}}\left[\frac{\mathrm{R}_{1}^{2}}{2 \mathrm{R}_{2}^{2}}+\frac{\mathrm{R}_{2}^{2}}{2 \mathrm{R}_{1}^{1}}-\sum_{\mathrm{i}=1}^{3} \alpha_{1 \mathrm{i}} \alpha_{2 \mathrm{i}}\right]}$

-gradient of the velocity of the liquid medium:

$$
\delta=\sqrt{\sum_{\mathrm{i}=1}^{3}\left(\frac{\partial \mathrm{W}_{\mathrm{oi}}}{\partial \mathrm{x}_{\mathrm{i}}}\right)^{2}=\sqrt{\frac{2 \mathrm{Q}^{2}}{\mathrm{R}_{1}^{3} \mathrm{R}_{2}^{3}}\left[\frac{\mathrm{R}_{2}^{3}}{\mathrm{R}_{1}^{3}} \sum_{\mathrm{i}=1}^{3} \alpha_{1 \mathrm{i}}^{4}+\frac{\mathrm{R}_{1}^{3}}{\mathrm{R}_{2}^{3}} \sum_{\mathrm{i}=1}^{3} \alpha_{2 \mathrm{i}}^{4}+\frac{\mathrm{q}^{2}}{3}-2 \sum_{\mathrm{i}=1}^{3} \alpha_{1 \mathrm{i}}^{2} \alpha_{2 \mathrm{i}}^{2}+6 \mathrm{q}\left(\mathrm{R}_{2}^{3}-\mathrm{R}_{1}^{3}\right)\right]}}
$$

Where $\mathrm{q}=\frac{\mathrm{R}_{2}^{3}-\mathrm{R}_{1}^{3}}{\mathrm{R}_{1}^{3} \mathrm{R}_{2}^{3}}$.

If the square of the velocity gradient $\delta^{2}$ is divided by the square of the velocity $\mathrm{W}_{\mathrm{o}}^{2}$, then this ratio will be equal to $\delta^{2} / \mathrm{W}_{\mathrm{o}}^{2}=4,5 \cdot \mathrm{S}$ where the symbol $\mathrm{S}$ denotes the next value

$$
\mathrm{S}=\frac{1}{\mathrm{R}_{1} \mathrm{R}_{2}} \cdot \frac{\frac{\mathrm{q}}{3} \mathrm{R}_{1}^{3} \mathrm{R}_{2}^{3}+\frac{\mathrm{R}_{2}^{3}}{\mathrm{R}_{1}^{3} \sum_{\mathrm{i}=1}^{3} \alpha_{1 \mathrm{i}}^{4}+\frac{2}{3} \mathrm{q}\left(\mathrm{R}_{2}^{3}-\mathrm{R}_{1}^{3}\right) \sum_{\mathrm{i}=1}^{3} \alpha_{1 \mathrm{i}}^{2} \alpha_{2 i}^{3}}}{\frac{\mathrm{R}_{2}^{2}}{2 \mathrm{R}_{1}^{2}}+\frac{\mathrm{R}_{1}^{2}}{2 \mathrm{R}_{2}^{2}}-\sum_{\mathrm{i}=1}^{3} \alpha_{1 \mathrm{i}} \alpha_{2 i}}
$$

We use the values $\mathrm{W}_{\mathrm{o}}^{2}, \delta^{2}$ for calculating the energy, which in the process of filtration currents is converted into the heat of a homogeneous fluid. It is composed of energy losses $\mathrm{E}_{\delta}$ due to a change in the rate of deformation of the flow and from energy losses $\mathrm{E}_{\mathrm{s}}$ due to the viscous friction of the liquid against the surface of the particle. Energy $E_{s}$ is equal to the product of the drag on the flow velocity $\mathrm{W}_{\mathrm{o}}$ of a homogeneous fluid:

$$
\mathrm{E}_{\mathrm{s}}=4,5 \eta_{\mathrm{o}} \frac{\mathrm{W}_{\mathrm{o}}^{2} \mathrm{~N}}{\mathrm{r}^{2}} \phi
$$

Here $\mathrm{N}$ is the number of particles per unit volume.

The energy losses due to the strain rate will be expressed in terms of the fluid velocity gradient

$$
\mathrm{E}_{\delta}=2 \eta_{\mathrm{o}} \delta^{2}
$$

The total energy loss is thus represented in the form of a sum

$$
\mathrm{E}=\mathrm{E}_{\delta}+\mathrm{E}_{\mathrm{s}}=2 \eta_{\mathrm{o}} \delta^{2}\left(1+\frac{\phi}{\Phi}\right) .
$$

In the last equality we took into account that $\Phi=2 \mathrm{r}^{2} \mathrm{~S}$ and $\mathrm{W}_{0}^{2}=\delta^{2} /(4,5 \cdot \mathrm{S})$.

Taking into account that the velocity $\overrightarrow{\mathrm{u}}_{\mathrm{o}}$ of a homogeneous fluid is related to the actual velocity $\overrightarrow{\mathrm{u}}$ of the inhomogeneous medium by the relation

We get

$$
\overrightarrow{\mathrm{u}}=(1-\phi) \overrightarrow{\mathrm{u}}_{\mathrm{o}} \text {, }
$$

$$
\mathrm{E}=2 \eta_{\mathrm{o}} \delta^{2}\left(1+\frac{\phi}{\Phi}\right) /(1-\phi)^{2}
$$

It follows from the last equality that the dynamic viscosity coefficient of a disperse medium $\eta(\phi)$ is determined by the following formula:

$$
\eta(\phi)=\eta_{\mathrm{o}}\left(1+\frac{\phi}{\Phi}\right) /(1-\phi)^{2}
$$


Formula (3) becomes Einstein's formula for the viscosity of the current inhomogeneous medium with particles of spherical shape at. In fact, for a uniform flow, the flow velocity will coincide with the tangential velocity of the streamlined body. If the body is a streamlined body, then the required velocity will be equal to the velocity at the point of tangency of the sphere lying at the end of the diameter drawn perpendicular to the direction of motion. In this case

$$
\mathrm{R}_{1}=\mathrm{R}_{2}=\mathrm{r}, \alpha_{11}=\alpha_{12}=\sqrt{2} / 2, \alpha_{21}=\alpha_{22}=-\sqrt{2} / 2 .
$$

Substituting these values into the formula for the form factor, we obtain $\Phi=2 r^{2} S=2$.

\section{Acoustic viscometer of inhomogeneous medium}

To determine the coefficients of viscosity and filtration of inhomogeneous media, acoustic viscometers are practically not used [6]. Meanwhile, they can be a very convenient means of field testing of the filtration characteristics of different media as applied to the refined definition of the parameters of the dynamic effects of groundwater flows on hydro constructions at the stages of preparation and formulation of design specifications [8].

The theory of acoustic viscometers is based on the system of hydrodynamic equations for inhomogeneous media. The system of hydrodynamic equations includes the equations of continuity, motion and state. Concerning the equation of continuity, it is necessary to assume that the entire volume occupied by the inhomogeneous medium is equal to $\bar{v}$; The volume occupied by the inhomogeneities is assumed to be equal to $\mathrm{v}_{1}$ the volume of the homogeneous medium $v_{0}$. Then the density $\bar{\rho}$ of the inhomogeneous system can be written as

$$
\bar{\rho}=\rho_{\mathrm{o}}+\frac{\mathrm{v}_{1}}{\mathrm{v}_{\mathrm{o}}}\left(\rho_{1}-\rho_{\mathrm{o}}\right)=\rho_{\mathrm{o}}(1-\phi)+\phi \rho_{1}
$$

Where $\phi=\mathrm{v}_{1} / \mathrm{v}_{\mathrm{o}}$ the concentration of inhomogeneities, the indices 0,1 refer, respectively, to a homogeneous medium and inhomogeneities. The equation of continuity can be made up of the following considerations. The amount of substance accumulated over time by convective transfer is equated to the change in mass in the volume under consideration. As a result, we will have:

$$
\int \frac{\partial}{\partial \mathrm{t}} \rho_{\mathrm{o}} \mathrm{md}{ }^{3} \mathrm{xdt}+\int \operatorname{div} \rho_{\mathrm{o}} \overline{\mathrm{u}} \mathrm{d}^{3} \mathrm{xdt}=0
$$

Here $\mathrm{m}$ denotes the quantity $(1-\phi), \overrightarrow{\mathrm{u}}$ - the real velocity of the inhomogeneous medium is analogous in sense of the filtration speed in hydrodynamics of porous media [10]. Suppose that this equality holds for any volume and that the number of particles is invariant, then the equation of continuity will look like this:

$$
\mathrm{m} \frac{\partial \rho_{\mathrm{o}}}{\partial \mathrm{t}}+\partial \mathrm{iv} \rho_{\mathrm{o}} \overrightarrow{\mathrm{u}}=0
$$

The formula for the viscosity of disperse media allows, according to the usual rule, to form a tensor of viscous stresses and to write the equation of motion of groundwater in the form

$$
\left.\bar{\rho} \frac{\partial \overline{\mathrm{u}}}{\partial \mathrm{t}}=-\nabla \overline{\mathrm{p}}+\eta(\phi) \Delta \overrightarrow{\mathrm{u}}+\frac{\eta(\phi}{3}\right) \nabla \operatorname{div} \overrightarrow{\mathrm{u}}
$$

To solve the hydrodynamic problems of disperse media, the equation of motion is supplemented by the continuity equation and the equation of state

$$
\frac{\mathrm{d} \overline{\mathrm{p}}}{\mathrm{dn}}=\frac{\mathrm{df}}{\mathrm{d} \bar{\rho}} \cdot \frac{\mathrm{d} \bar{\rho}}{\mathrm{dn}}=\mathrm{c}_{*}^{2} \frac{\mathrm{d} \bar{\rho}}{\mathrm{dn}}
$$

Where $\overline{\mathrm{c}}_{*}=\sqrt{\mathrm{d \overline { \textrm {p } } / \mathrm { d } \overline { \rho }}}$ - is the sound velocity of an inhomogeneous medium. 
Equations (4), (5), (6) are sufficient to solve the acoustic problem and to justify the acoustic viscometer. If we assume that during the transition through the acoustic front the continuity gap is sustained not by the physical-mechanical quantities entering the system of equations of hydrodynamics such as density, pressure and velocity, but only their spacetime derivatives of the first and second order, then the written equations can be applied to the apparatus Hadamard-Predvoditeleva [11]. Using this apparatus, we obtain the following expression for the complex velocity of ultrasound propagation:

$$
\overline{\mathrm{c}}^{2}=\overline{\mathrm{c}}_{*}^{2}+\mathrm{i} \frac{\eta(\phi) \omega}{\bar{\rho} \overline{\mathrm{g}}_{1}} \overline{\mathrm{c}}
$$

Here $\bar{c}_{1}$ denotes the real part of the complex velocity, $\omega=2 \pi v$ the frequency of the ultrasonic sound. Selecting the imaginary and real parts, we find the following relations for the velocity and the absorption coefficient $\alpha$ :

$$
\begin{array}{r}
\overline{\mathrm{c}}_{1}=\overline{\mathrm{c}}_{*} \\
\alpha=\frac{2}{3} \frac{\eta(\phi) \omega^{2}}{\bar{\rho} \overline{\mathrm{c}}_{*}^{3}}
\end{array}
$$

If we assign the obtained absorption coefficient to the absorption coefficient for a medium that does not contain particles, then we have:

$$
\frac{\alpha}{\alpha_{\mathrm{o}}}=\frac{(1+\phi / \Phi)}{(1-\phi)^{2}} \frac{\rho_{\mathrm{o}} \mathrm{c}_{\mathrm{o}}^{3}}{\bar{\rho} \overline{\mathrm{c}}^{3}}
$$

To evaluate the capabilities of the acoustic viscometer, comparative experiments were conducted to determine the viscosity with traditional Ostwald flow-through capillary viscometers. As a non-uniform medium, two-phase media consisting of particles of lycopodium in salted water were used.

The particle radius was $8 \mu \mathrm{m}$, the volume concentration $\phi=15 \%$, the water temperature $\mathrm{T}=21^{\circ} \mathrm{C}$, the ratio of the wavelength of the ultrasonic oscillations to the particle radius was determined by the condition that corresponded to the Stokes acoustic dispersion mode with the proportionality of the absorption coefficient to the square of the ultrasound frequency. The ultrasound velocity was measured by the interferometric method, the absorption coefficient by the diffraction method [12]. Data on the absorption coefficient measurements and calculations with viscosity according to formulas with different shape

\begin{tabular}{|c|c|c|c|c|c|c|}
\hline $\begin{array}{c}\text { v, } \\
\text { Mhz }\end{array}$ & $\begin{array}{c}\text { Exper } \\
\text { iment } \\
\alpha, \\
\text { cm-1 }\end{array}$ & $\begin{array}{c}\text { Experiment } \\
\alpha / v^{2} \cdot 10^{-17} \\
\text { s2/cm }\end{array}$ & $\begin{array}{c}\text { Calcula } \\
\text { tion of } \\
(1) \\
\alpha, \mathrm{cm}- \\
1 \\
\end{array}$ & $\begin{array}{l}\text { Calculation of } \\
\text { (1) } \\
\alpha / v^{2} \cdot 10^{-17} \mathbf{s} 2 / \mathbf{c} \\
\mathbf{m}\end{array}$ & $\begin{array}{c}\text { Calcula } \\
\text { tion of } \\
(1) \\
\alpha, \text { cm- } \\
1 \\
\end{array}$ & $\begin{array}{c}\begin{array}{c}\text { Calculation of } \\
(\mathbf{3}) \\
\alpha / v^{2} \cdot 10^{-17} \mathbf{s} 2 / \mathbf{c} \\
\mathbf{m}\end{array}\end{array}$ \\
\hline 10,2 & 0,220 & 2,115 & 0,160 & 1,538 & 0,226 & 2,172 \\
\hline 11,7 & 0,340 & 2,484 & 0,220 & 1,607 & 0,297 & 2,170 \\
\hline 14,2 & 0,480 & 2,380 & 0,320 & 1,587 & 0,438 & 2,172 \\
\hline 15,7 & 0,570 & 2,312 & 0,380 & 1,542 & 0,535 & 2,170 \\
\hline 19,3 & 0,760 & 2,040 & 0,580 & 1,557 & 0,810 & 2,175 \\
\hline 20,9 & 0,830 & 1,900 & 0,680 & 1,557 & 0,950 & 2,175 \\
\hline 21,7 & 1,150 & 2,442 & 0,730 & 1,550 & 1,020 & 2,166 \\
\hline 22,9 & 1,160 & 2,212 & 0,820 & 1,564 & 1,140 & 2,174 \\
\hline
\end{tabular}
factors are given in Table 1 and in Fig. 1.

Table 1. Absorption coefficient in two-phase medium of lycopodium in water at various ultrasound frequencies. 


\begin{tabular}{|c|c|c|c|c|c|c|}
\hline 29,0 & 1,680 & 1,998 & 1,310 & 1,558 & 1,830 & 2,176 \\
\hline & & $\mathbf{2 , 2}$ & & $\mathbf{1 , 6}$ & & $\mathbf{2 , 2}$ \\
\hline
\end{tabular}

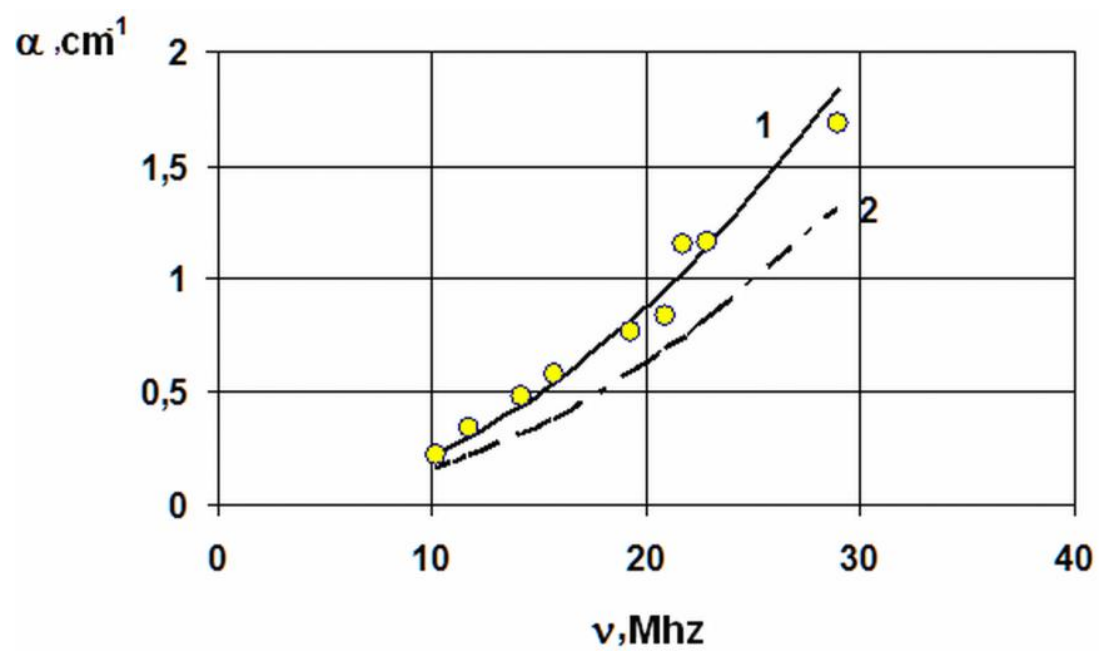

Fig. 1. Absorption coefficient in the two-phase system of lycopodium-water. Mugs - experimental data of the authors; 1 - calculation according to formula (8) with viscosity at form factor $\Phi=2 r^{2} S=0,03 ; 2$ - calculation by formula (8) with viscosity at $\Phi=2 r^{2} S=2$.

The value of the form factor $\Phi$, according to formula (1) for the viscosity of two-phase media, corresponds to the assumption of spherically symmetric flow of particles in the process of percolation through the ground. In calculations with a relatively low value of the form factor, it means that in filtration the point at which the flow velocity is equal to the velocity of the incoming flow does not coincide with the surface of the particle. In our case, this point should be outside the particle at a distance of $16 \mu \mathrm{m}$ from its center. This distance is less than the distance between the particles between the particles, which, for example, with a cubic arrangement of particles is $270 \mu \mathrm{m}$. It is not excluded that this circumstance can lead to discrepancies in the measurement data with the use of acoustic viscometers and data obtained by measurements with a flowing capillary viscometer. The performed viscosity measurements in the Ostwald viscometer have shown that the viscosity of the investigated inhomogeneous medium with particles of a non-ideal form has the value $4.7 \cdot 10^{-2} \mathrm{pz}$, which agrees with the data on an acoustic viscometer, which according to our measurements give a viscosity value of $4.5 \cdot 10^{-2} \mathrm{pz}$. With a shape factor $2 \mathrm{r}^{2} \mathrm{~S}=2$ characteristic of ideal spherical particles, the coefficient of viscosity corresponding to our acoustic measurements was $1.12 \times 10^{-2} \mathrm{pz}$. Comparison of the measurement data of the viscosity of inhomogeneous media obtained by acoustic and capillary viscometers leads to the conclusion that in non-idealistic movements a non-ideal particle shape corresponding to the Poiseuille character of the flow of a two-phase medium is manifested.

At the same time, there remains the question of the calculation of the form factor independent of acoustic measurements and its solution requires separate consideration. According to Einstein's formula (1), the value of the form factor is determined by the flow conditions of solid particles by the current of the liquid medium in which they are located. The nature of the flow around depends on the concentration of particles in the liquid. An analysis of the results of measuring the absorption coefficient of ultrasound in the twophase medium studied by us (a particle of lycopodium in water) showed that the absorption increases with the concentration of particles linearly. This character of the dependence of 
absorption with the concentration is described by formula (8) on the assumption that the shape factor varies according to the law:

$$
\Phi=2 r^{2} \mathrm{~S}=(28+11 \phi) \cdot 10^{-3} .
$$

The obtained results showed the identity of the measurement data of the acoustic and capillary viscometers in the region of the Stokes dispersion with a quadratic dependence on the frequency of ultrasonic vibrations. On the basis of the results of many experiments carried out with various two-phase media, it is permissible to conclude that in the calculation of the dynamic parameters of groundwater filtration currents, a generalized hydrodynamic formula for the viscosity of two-phase media can be used that takes into account the nature of particles of different configurations flowing through the liquid medium.

\section{Impacts of viscous groundwater currents on hydraulic structures}

The tasks of non-pressure filtration flow of groundwater are of practical interest in the calculation of dynamic loads of hydraulic origin on various elements of hydraulic structures [13]. When the groundwater is stationary, their free surface is horizontal and the pressure on the structures is determined by the hydrostatic head. During the filtration motion, the free surface changes, decreasing along the flow. The contribution of the high-pressure head of the groundwater, depending on the viscosity of the inhomogeneous medium, creates additional loads on the foundations of the structures. The difficulties in obtaining an exact solution to the problems of non-pressure filtration by the definition of depressive changes in heads are associated with the uncertainty of the shape of the region occupied by the ground flow and the absence of relationships that take into account the influence on the filtration parameters of the head loss due to viscosity. The results presented in the previous sections of the paper on the calculation of the viscosity of inhomogeneous media make it possible to introduce refinements in the solution of the problem of determining the effect of viscous filtration currents on dam-free vacuum weirs of a practical profile. As a design object of the hydraulic engineering structure, the spillway of the waterworks has been chosen. The area of the underground filtration currents under the dam is presented in the diagram of Figure 2. The filtration in the base of the hydro constructions occurs under the action of the head $\mathrm{h}$, equal to the difference in the water levels in the upper pool and the downstream.

$$
\mathrm{h}=\nabla \mathrm{b}-\nabla_{\mathrm{H}} \text {. }
$$

We will assume that the dam's flute, all along including the drain, the water-draining and the water-pipe are waterproof, and the outlet of the filtration flow into the lower tail is carried out through the holes in the apron. The characteristics of the filtration flow in any of its zones of the underground contour of the hydro construction can be determined using a hydrodynamic grid. 


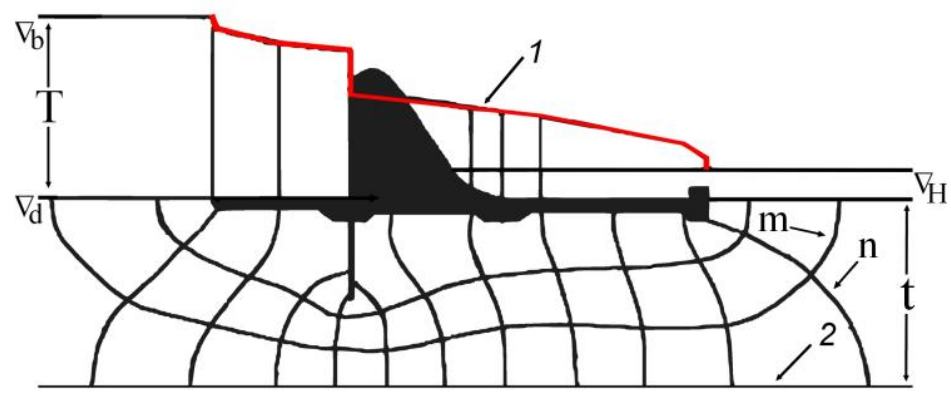

Fig. 2. Calculation scheme for determining the dynamic effect of the filtering flow of groundwater on a weir of a practical profile: 1 - piezometric line, 2- waterproof.

The hydrodynamic grid consists of current lines and equipotentials (lines of equal heads). The entire filtration area is divided by lines of constant heads into n-lines, and current lines to m-belts. The lines of current to the dam of the dam fit at right angles, dividing the distance between the foundation of the dam and the water column to different parts. Equipotential lines to the sections of the flux cross the intermediate lines of current and waterproof at right angles. As a result of the intersections of the current lines and equipotentials, curvilinear quadrangles with straight angles and approximately the same average lines are obtained. The whole filtration area is thus divided by equipotentials into n-bands, and current lines by m-belts. The acting head determines the specific mechanical energy that is expended to overcome the viscous resistance along the whole path of the flow of the filtration flow. The value of the static head $\mathrm{T}$ on the weir (the depth of the stream in front of the weir) is read as the difference between the marks of the upper tail and the bottom:

$$
\mathrm{T}=\nabla \mathrm{b}-\nabla \mathrm{d} \text {. }
$$

The loss of pressure between adjacent lines of equipotentials is:

$$
\Delta \mathrm{h}=\mathrm{h} / \mathrm{h} \text {, }
$$

Where $\mathrm{h}=\nabla \mathrm{b}-\nabla_{\mathrm{H}}$ is the difference between the marks of the upper and lower tunnels, $\mathrm{n}$ the number of bands between equipotentials.

The rate of filtration flow during laminar filtration is calculated from the Darcy law dependence:

$$
\mathrm{u}=-\mathrm{k}_{\phi} \mathrm{J}=-\mathrm{k}_{\phi} \frac{\Delta \mathrm{z}}{\Delta \mathrm{x}}=-\mathrm{k}_{\phi} \frac{\mathrm{t}}{\sqrt{\mathrm{k}_{\mathrm{p}}}} .
$$

Where $\mathrm{k}_{\phi}$ is the filtration coefficient; $\mathrm{J}=\Delta \mathrm{z} / \Delta \mathrm{x}$ - hydraulic slope; $\Delta \mathrm{z}=\mathrm{t}$ is the thickness of the filtration layer, $\Delta \mathrm{x}=\sqrt{\mathrm{k}_{\mathrm{p}}}$ is the length of the current line segment between equipotentials, expressed in terms of the soil permeability coefficient $\mathrm{k}_{\mathrm{p}}$.

Calculation of the force action of the filtration flow on the dam of the dam should be carried out proceeding from the static and high-speed head in any section of the underground circuit. To determine the pressure of the filtration flow on the base of the water-drain, a graphic method for constructing a piezometric line reflecting the hydraulic action of groundwater can be used. For this purpose, vertical lines are drawn on the current line, which coincides with the waterproof contour of the structure at the points of intersection of equipotentials with the underground contour of the structure. On them upwards from the horizontal axis $\mathrm{OX}$, starting from the initial point coinciding with the bottom, the values of the total head $\mathrm{H}_{\mathrm{i}}$ at $\mathrm{i}=1,2 \ldots \mathrm{n}$ are displayed:

$$
\mathrm{H}_{\mathrm{i}}=\mathrm{T}-\mathrm{i} \Delta \mathrm{h} \text {, }
$$


corresponding to the values of each equipotential, where the loss of the pressure of the filtration flow between equipotentials is equal to

$$
\mathrm{H}_{\mathrm{i}}=\mathrm{T}-\left(\frac{\mathrm{h}+\frac{\mathrm{u}^{2}}{2 \mathrm{~g}}}{\mathrm{n}}\right) \mathrm{i}
$$

Here $h=\nabla_{B}-\nabla_{H}$-static pressure, u-velocity of filtration flow.

In view of Darcy's law, for the sum of the static and high-speed heads in the movement of groundwater, we have

$$
\Delta \mathrm{h}_{\mathrm{i}}=\left(\frac{\mathrm{h}+\frac{\mathrm{k}_{\Phi}{ }^{2}}{2 \mathrm{~g}}\left(\frac{\mathrm{dz}}{\mathrm{dx}}\right)^{2}}{\mathrm{n}}\right) \mathrm{i}=\left(\frac{\mathrm{h}+\frac{\mathrm{k}_{\Phi}^{2}}{2 \mathrm{~g}}\left(\frac{\mathrm{t}}{\sqrt{\mathrm{k}_{\mathrm{p}}}}\right)^{2}}{\mathrm{n}}\right) \mathrm{i}
$$

Expressing the coefficient of filtration, through the coefficient of permeability

$$
\mathrm{k}_{\Phi}=\frac{\mathrm{k}_{\mathrm{p}} \bar{\rho} \mathrm{g}}{\bar{\eta}(\phi)}
$$

we can rewrite the last expression in the following form:

$$
\Delta \mathrm{h}_{\mathrm{i}}=\left(\frac{\mathrm{h}+\frac{\mathrm{k}_{\Phi} \overline{\bar{\rho}} \mathrm{t}^{2}}{2 \bar{\eta}(\phi)}}{\mathrm{n}}\right) \mathrm{i}
$$

As a result, the calculation formula (16) for constructing the line of the hydraulic head of groundwater on the foundation of the hydraulic structure takes the following form:

$$
\mathrm{H}_{\mathrm{i}}=\mathrm{T}-\left(\frac{\mathrm{H}+\frac{\mathrm{k}_{\Phi} \bar{\rho} \mathrm{t}^{2}}{2 \bar{\eta}(\phi)}}{\mathrm{n}}\right) \mathrm{i}
$$

The shape of the piezometric line is obtained by plotting upward values from the horizontal axis of the foundation of the dam from the initial point of the cord to the apron. different parts of the current line, which coincides with the waterproof circuit of the structure. On the water piezometric line above the water level in the downstream and this back pressure must be compensated for by the weight (thickness) of the water plate. The pressure at any point of the underground drain is as follows:

$$
\mathrm{P}=\bar{\rho} \mathrm{g}(\mathrm{H}+\mathrm{y}) \text {, }
$$

where the current $\mathrm{h}$ is the head, $\mathrm{y}$ is the penetration of the point under consideration to the bottom level. As follows from formula (19), the diagram of hydrodynamic pressure is composed of two diagrams: the piezometric line and the line of deepening the points of the contour of the flute. The total pressure at any of the sections of the subterranean contour is expressed by the area of the part of the pressure diagram corresponding to this section multiplied by the specific gravity of the water. The pressure force $F_{B}$ of the filtration flow per one running meter of the weir is

$$
\mathrm{F}_{\mathrm{b}}=\bar{\rho} \mathrm{g} \sigma_{\mathrm{b}},
$$


Where $\sigma_{b}=\sum_{i=1}^{2} \frac{H_{i}}{2} \Delta L$ - the area of the diagrams of the heads at the beginning $H_{i}$ and the end $\mathrm{H}_{\mathrm{i}+1}$ of the sole of the water-discharge length $\Delta \mathrm{L}$. The pressure of the filtration flow to the entire weir is equal to:

$$
\mathrm{F}=\mathrm{F}_{\mathrm{b}} \mathrm{B}
$$

Here $\mathrm{B}$ is the width of the stream in front of the weir.

As an analysis of the relationships obtained, calculations of hydraulic loads on the sections of the weir foundation of a practical profile are performed, depending on a twophase medium consisting of solid particles of different shapes in the water of their concentration and viscosity.

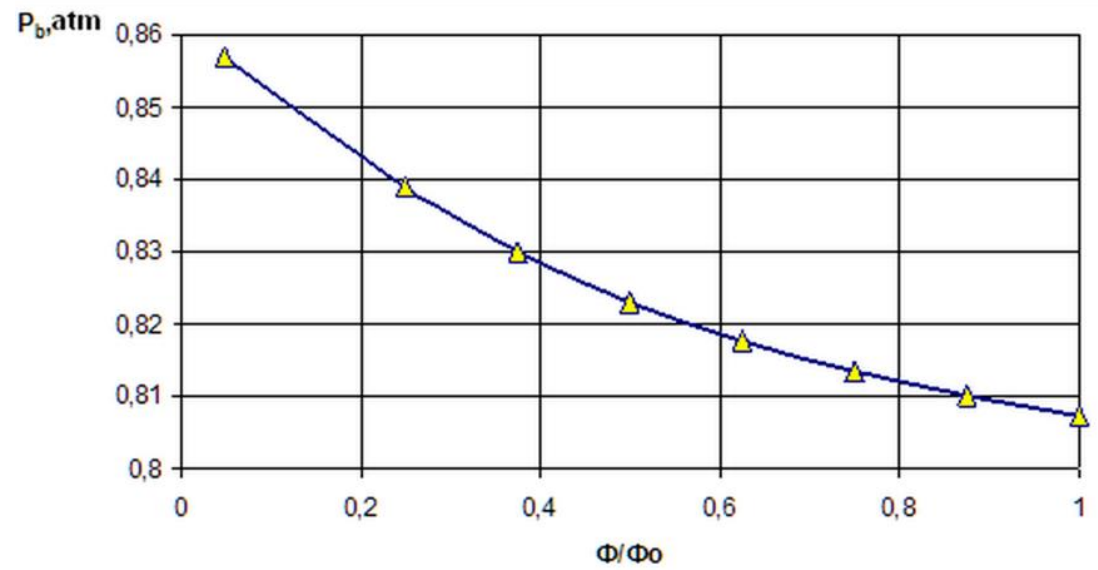

Fig. 3. Relative contribution of the velocity head to the pressure $\mathrm{Pb}$ on the bottom of the waterdraining dam of the groundwater filtration flow with particles having a factor of form $\Phi=r^{2} S$ normalized to the value $\Phi_{0}=2$ corresponding to the spherical configuration.

The results of calculations of the relative contribution of the velocity head to the total pressure of the non-pressure filtration flow in the underground contour of the hydro structure indicate a significant dependence on the shape of the particles. The relative contribution of the velocity head decreases when passing to filtration through a medium of a containing particle with an ideally spherical configuration (see Fig. 3).

With an increase in the effective viscosity of groundwater, there is a sharp decrease in the force load on the flute of the spillway dam (Fig. 4 (a)).
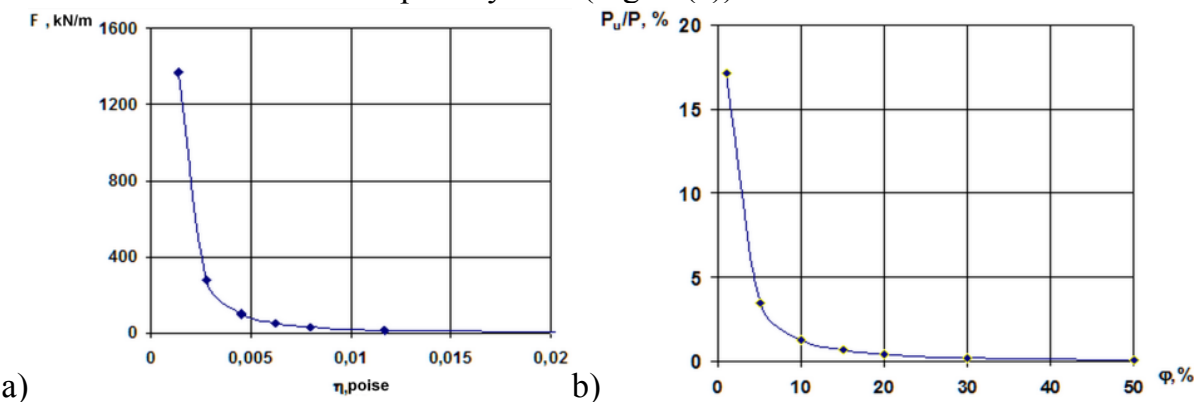

Fig. 4. a) Effort force $F$ of groundwater of different viscosity $\bar{\eta}$ per running meter of the flux of the weir. The calculation is performed for the degree of deviation of the particles from the spherical shape $\Phi=/ \Phi_{0}=0,16$. b) The relative contribution of the velocity head Pu to the pressure P on the bottom of the water-draining dam of the filtration flow of groundwater, depending on the concentration of the inhomogeneous medium. 
The effect of the concentration of groundwater particles on the dynamic effect of filtration flow on the base of the hydro construction is clearly manifested with relatively low values of the particle density (Fig. 4 (b)). As a percentage of the relative contribution of the velocity head $\mathrm{Pu}$ to the total pressure, it reaches values of the order of $20 \%$, mainly for particles of irregular shape, characterized by relatively low values of the shape factor.

\section{Conclusions}

Based on the results of an analysis of the dynamic effects of the effective viscosity of inhomogeneous media on elements of hydro constructions, the following conclusion can be drawn. In calculations of groundwater pressures in the case of filtration without pressure on the elements of hydraulic structures, along with hydrostatic effects, take into account the dynamic effects of filtration flows, especially when particles with configurations other than spherical shape and relatively low concentrations are present in an inhomogeneous medium. Errors in assessing the levels of permissible hydraulic loads on the foundations of hydro constructions can reach values of the order of a tenth of a percent. The proposed formulas for determining the effective viscosity of inhomogeneous media and the method for estimating the piezometric loads on hydro structures can be recommended in the practical design of hydrotechnical construction sites.

This research work was carried out within the framework of the State task (No. 0149-2018-0001; No.AAAA-A16-116032810088-8)

\section{References}

1. Bear J., Dynamics of fluids in porous media (Courier Corporation, 2013)

2. Einstein A., Ann. Phys, 19, 2. (1906)

3. Eilers von H. Kolloid-Zeitschrift, 97, 3 (1941)

4. Graham A.L., Appl. Sci. Res, 37, 3-4. (1981)

5. Basniev KS, Kochina I.H., Maksimov B.M. Underground hydromechanics (Moscow: Nedra, 1993)

6. Thurston R. Physical acoustics, vol. 1 (Part A) (ed. U. Mason, 1966)

7. Bear J. Hydraulics of groundwater (Courier Corporation, 2012)

8. SNiP 11-02-96. Engineering surveys for construction. Basic provisions (Moscow: Gosstroy of Russia, 1997)

9. Polubarinova-Koch P.I. Theory of ground water movement (Princeton University Press, 2015)

10. Nigmatulin RI, Soloviev DA Fundamentals of hydromechanics (Moscow: Litterra, 2012)

11. Predvoditelev A.S. Mechanics of movements (Minsk: Publishing House of the Belarusian State University, 1975)

12. Shebalkova L.V., Legkiy V.N., Romodin V.B. Microwave and ultrasonic sensors. (Novosibirsk: NSTU, 2015)

13. Solovyev A.A., Solovyev D.A., Shilova L.A. Vestn. MGSU 13, 7 (118) (2018) DOI: 10.22227/1997-0935.2018.7.885-891 